\title{
Analytical model of the effect of misfit dislocation character on the bubble-to-void transition in metals
}

\author{
Enrique Martínez, ${ }^{1, *}$ D. Schwen, ${ }^{2}$ J. Hetherly, ${ }^{1}$ and A. Caro ${ }^{1}$ \\ ${ }^{1}$ Material Science and Technology Division, MST-8, \\ Los Alamos National Laboratory, Los Alamos, 87545 NM, USA \\ ${ }^{2}$ Fuels Modeling and Simulation, Idaho National Laboratory, Idaho Falls, ID 83415
}

\begin{abstract}
This paper addresses the role of misfit dislocations in the nucleation and growth of nanoscale $\mathrm{He}$ bubbles at interfaces. In a recent work, we studied the nanoscale effects on the capillarity equation and on equilibrium conditions. We proposed an expression for surface energy and for the equation of state, EOS, for He in bubbles, which have a size dependence that captures the role of the interface forces, which become relevant at the nanoscale. Here we determine the EOS for several twist grain boundaries in $\mathrm{Fe}$ and $\mathrm{Cu}$ and incorporate these results into the rate equation that determines the bubble-to-void transition, focusing on the influence of interface dislocations on the evaporation rate of vacancies. We find a significant effect of the magnitude of the Burgers vector of the dislocations on the critical radius for the transition. These results give a quantitative way to characterize grain boundaries in their ability to capture He and alter the onset of swelling.
\end{abstract}

PACS numbers:

Keywords: Segregation, Diffusion, Irradiation

\section{INTRODUCTION}

Helium appears in fusion and fission nuclear materials as a result of nuclear reactions. Its presence is usually a nuisance as it affects mechanical properties in dramatic ways. Chemically inert, He has an exceedingly low solubility in all metals and a strong tendency to precipitate heterogeneously at defects such as dislocations, grain boundaries, or interfaces, as is the case in ODS steels ${ }^{1}$. Both experiments and modeling have recently shown that the atomic structure of the interface affects the He bubble distribution at the interface and the void denuded zone around the boundary ${ }^{2-6}$. He bubbles and their influence on swelling have received significant attention from the nuclear materials science community for years. Historically, the 80 's was a decade when most of the analytic treatment of this problem, in terms of rate or diffusion equations, was developed. For a comprehensive treatment of the problem see Ref. 7. The time evolution of a bubble in a material under irradiation is determined by the balance between defects (interstitials, vacancies, and $\mathrm{He}$ ) arriving and escaping the evolving bubble. Since each one of these defects have their own properties (source, annihilation, recombination, and trapping at sinks terms) the resulting rate equations usually do not have a truly predictive power, but help at giving the type of effects that may occur for a reasonable choice of the numerous parameters entering the model.

One of the main conclusion of these theories was the existence of a critical radius for the bubble-to-void transition, that is, a critical size for a given He content, from which bubbles grow indefinitely by capturing vacancies and becoming voids. Another key discovery was the existence of a second radius, smaller than the critical, called stable radius, where bubbles remain at stationary size under irradiation. These two radii together determine a bimodal bubble size distribution, a fact verified experi- mentally.

The equation that displays these effects is ${ }^{7-12}$,

$$
\frac{d R}{d t}=\frac{\Omega}{R}\left[Z_{v}^{c} D_{v} C_{v}-Z_{i}^{c} D_{i} C_{i}-Z_{v}^{c} D_{v} C_{v}^{c}\right]
$$

where $R$ is the cavity radius, $\Omega$ is the volume of an atom, Z's are capture cross sections or sink strengths, proportional to the area of the cavity, D's are diffusion coefficients, C's are concentrations, $v$ stands for vacancies, $i$ for interstitials, and $c$ for cavity. The first term on the r.h.s represents the contribution to growth rate via vacancy capture, the second, with negative sign, represents interstitials capture; both depend on the concentration of defects in bulk far from the cavity, determined by the irradiation condition. The third term represents the evaporation of vacancies and depends on the concentration of vacancies at the surface of the cavity. This quantity is obtained assuming that in the matrix, but very close to the bubble surface, the vacancy concentration in the crystal is at equilibrium with the bubble. The equilibrium condition translates into the equality of the chemical potentials inside and outside the bubble. The chemical potential for vacancies in the lattice close to the cavity, $\mu_{v}^{L}$ at concentration $C_{v}^{c}$, and the chemical potential for vacancies at a bubble with radius $R$, gas pressure $P$, and surface energy $\gamma, \mu_{v}^{b}$, are given by Ref. 11,

$$
\begin{aligned}
& \mu_{v}^{L}=k T \ln \left(\frac{C_{v}^{c}}{C_{v}^{e}}\right) \\
& \mu_{v}^{b}=\Omega\left(\frac{2 \gamma}{R}-P\right)
\end{aligned}
$$

Equating them, we obtain:

$$
C_{v}^{c}=C_{v}^{e} \exp \left[\frac{\Omega}{k T}\left(\frac{2 \gamma}{R}-P\right)\right]
$$




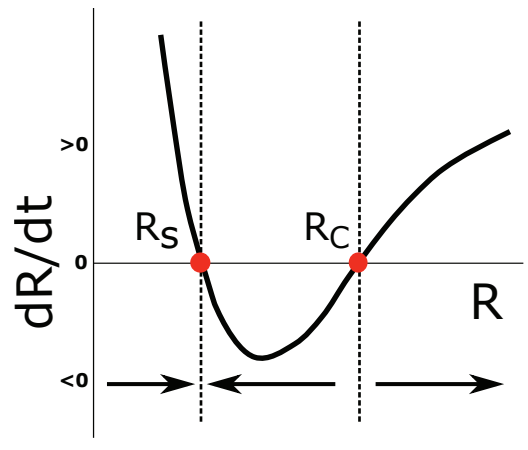

FIG. 1: Schematic view of the radial growth rate of a $\mathrm{He}$ bubble versus its radius in a situation of constant He content and irradiation produced flux of vacancies and interstitials. $R_{s}$ and $R_{c}$ are the stable and critical radii respectively.

with

$$
C_{v}^{e}=\exp \left[\frac{-\epsilon_{v}^{f}}{k T}\right]
$$

where $\mathrm{k}$ is the Boltzmann constant, and $\mathrm{T}$ the temperature. In the absence of irradiation, Eq. (3) tells that if the gas pressure $P$ equals the surface tension $2 \gamma / R$ then the bubble is in equilibrium with the crystal in the sense that there is no net vacancy flux, since their concentration at the surface of the bubble equals that in bulk.

The competition between out and incoming fluxes of defects opens the possibility to changes in sign in the bubble growth rate. For a judicious choice of parameters, and the simplifying assumptions of stationary vacancy and interstitial concentrations in bulk (which depend on the irradiation conditions) and of equilibrium concentration of vacancies at the surface of the cavity, i . e. $C_{v}^{c}$ given by Eq. (3), a possible solution to this equation is represented in Fig. 1, where the intersections of the curve with the x-axis determine the stable, $R_{s}$, and critical, $R_{c}$, radii. The arrows at the bottom of the figure indicate the stability of the points: departures from $R_{s}$ bring the bubble back to $R_{s}$, while deviations from $R_{c}$ bring the bubble to either infinite growth or to the stable radius.

For constant values of the first two terms in Eq. (1), the growth rate is determined by the equilibrium vacancy concentration at the surface of the cavity; for a value larger than the stationary concentration of vacancies in bulk, the bubble will evaporate vacancies, while for values less than bulk, it will absorb vacancies. Again, for a detailed derivation of these equations see Ref. 7 .

This paper studies the validity of this approach to heterogeneous bubbles that evolve at an interface characterized in terms of misfit dislocations.

\section{BACKGROUND}

We have heretofore presented the traditional formulation of the problem, developed in the 80's ${ }^{9,13}$. Equations
3 and 4 are the result of a continuum linear elastic approximation, namely, the properties of the gas are represented by $\mathrm{P}$, and the properties of the metal matrix, where the bubble is located, by $\gamma$, and the two terms give independent contribution to the energy involved in adding a vacancy to the cavity, neglecting any gas-surface interaction. This paper analyzes how $\mathrm{P}$ and $\gamma$ change for a given He density when the bubble grows at an interface that can be characterized in terms of misfit dislocations.

Recently, we have shown ${ }^{14,15}$ that the capillarity equation in its original form can not be used for fluid bubbles embedded in solids because it suffers from the same limitations discussed by Tolman ${ }^{16}$ for the case of a membrane separating two fluids. The fact that the interface has a finite width leads to diverse phenomena, such as the generation of a structure within the bubble that makes pressure an ill defined quantity, or the fact that the equilibrium condition does not happen for $P=2 \gamma / R$ but for an amount of He atoms that differs by more than a factor of two from it. We also determined that in the case of bubbles in solids, $P$ and the density $\rho$ can no longer be defined as global quantities related by an equation of state (EOS) for the fluid, but they become functions of position since the bubble develops a core-shell structure originated in the atomic scale nature of the interface. The range of the metal-gas interaction defines the width of the interface, that for the He-Fe system studied is $\sim 0.4 \mathrm{~nm}$, implying that bubbles around $1 \mathrm{~nm}$ in diameter are almost entirely affected by this interface effect, creating a radial pressure profile that is not constant in the fluid, nor zero in the metal, as the theory of the Eshelby inclusion would predict ${ }^{17}$.

By relating the average pressure to an average density we proposed a new EOS for the pressure in the bubble that depends on the bubble radius and could be used in models of equilibrium or kinetic processes. The purpose of this paper is to explore in detail these nanoscale effects for bubbles nucleated at different environments characterized by a dislocation structure, such as twist grain boundaries (TGB) in bcc Fe and fcc Cu. Ultimately, the question we want to answer is: what is the effect of the microstructure in the bubble-to-void transition? Specifically we aim at finding the dependence of the critical radius, a relevant parameter that determines the onset of swelling, in terms of the material and GB properties.

\section{RESULTS}

\section{A. Study of the bubble pressure at interfaces}

The fact that the He-metal interaction makes He atoms inside a bubble feel different forces depending on where in the bubble the atoms sit (close to the surface of the bubble, a strong metal repulsion creates forces that lower He density) has consequences when the bubble locates at a boundary composed of misfit dislocations: these density variations are sensitive to the presence of dislocations 


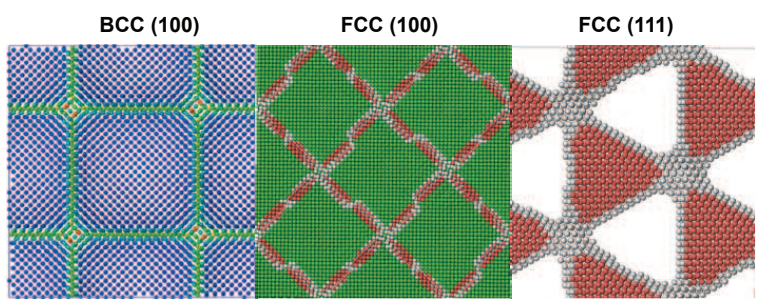

FIG. 2: Twist interfaces studied in this work. Left: $2^{\circ}$ bcc (100); Center: $4^{\circ}$ fcc (100); Right: $2^{\circ}$ fcc (111).

and translate into changes in the EOS of He. We derive these new EOSs and apply them to calculate the critical radius for the bubble-to-void transition.

To find the pressure dependence on bubble radius and location (i. e. bulk or dislocation cores in twist boundaries) that could be used in Eq. 4 to predict equilibrium or rate processes, we perform here a systematic evaluation of the EOS based on a similar methodology as in Ref. ${ }^{15}$. It consists of creating a spherical cavity of radius $R$ in a cubic metal sample with periodic boundary conditions and big enough to minimize bubble-bubble interactions, equilibrated at a temperature $T$, and filling it with a number of $\mathrm{He}$ atoms that we choose between 0.1 and 2 times the number of metal atoms removed (the unit of density is He/matrix atom vacancy). The sample is equilibrated and then the average pressure and volume are measured. The final result for each bubble is its radius, density, temperature and pressure. The values for several hundred $(R, \rho, T, P)$ points represent the raw data for the EOS. A non-linear fitting procedure determines the parameters in the expression we use for the EOS (Eq. 6). In this study we use a simpler and less accurate expression for the EOS than in our previous work $^{14}$, as we are presenting several EOSs for different environments and want to keep the numerical complexity low

$P(\rho, T, R)=\left(-1+e^{\left(c_{0}+c_{1} T\right) \rho}\right)\left(a_{0}+a_{1} T+\frac{b_{0}+b_{1} T}{R}\right)$

Six cases were studied, namely, pure bcc Fe and fcc $\mathrm{Cu}, 2^{\circ}\{100\}$ and $\{110\}$ TGBs in Fe, and, $4^{\circ}\{100\}$ and $2^{\circ}\{111\}$ TGBs in Cu. Figure 2 shows the dislocation structure for three of these interfaces. In all TGB cases, semi-grand canonical Monte Carlo simulations show that He segregates to the dislocation intersections ${ }^{4}$. The results of the fittings for the EOS of He at these interfaces are given in Table I.

To analyze this data in terms of the dependence of the bubble pressure on its size and location, let us focus in particular on the cases at density $\rho=1 \mathrm{He} / \mathrm{Vac}$ and $T=600 \mathrm{~K}$. Figure 3 shows the results for $\mathrm{Cu}$, for bulk and two twist boundaries, $\{100\}$ and $\{111\}$ (Fig. 3(a)), composed of screw dislocations with different Burger vectors. It also displays the values for bulk $\mathrm{Fe}$ and a $\{100\}$ TGB (Fig. 3(b)). The first observation is that, at a given density, the pressure has a significant dependence on the
TABLE I: Parameters for the simplified version of the EOS of He bubbles in metals, Eq (6).

\begin{tabular}{|c|c|c|}
\hline Parameter & Fit & Standard error \\
\hline$a_{0}$ & 0.7502 & 0.12 \\
\hline$a_{1}$ & 0.00095 & 0.0002 \\
\hline$c_{0}$ & 1.602 & 0.046 \\
\hline$c_{1}$ & -0.000150 & 0.000065 \\
\hline Bulk Fe & & \\
\hline$b_{0}$ & 0.742 & 0.029 \\
\hline$b_{1}$ & 0.000101 & 0.000048 \\
\hline (100) Twist Fe & & \\
\hline$b_{0}$ & 0.767 & 0.027 \\
\hline$b_{1}$ & 0.000075 & 0.000045 \\
\hline Bulk Cu & & \\
\hline$b_{0}$ & 0.685 & 0.036 \\
\hline$b_{1}$ & -0.000016 & 0.000063 \\
\hline (100) Twist $\mathrm{Cu}$ & & \\
\hline$b_{0}$ & 0.602 & 0.042 \\
\hline$b_{1}$ & -0.000085 & 0.000070 \\
\hline (111) Twist $\mathrm{Cu}$ & & \\
\hline$b_{0}$ & 0.613 & 0.042 \\
\hline$b_{1}$ & -0.000118 & 0.000072 \\
\hline
\end{tabular}

radius of the bubble for radii $\sim 2 \mathrm{~nm}$ or less, as already discussed in our previous work ${ }^{14,15}$, and the second observation is that bubbles growing at the interface may show a small difference in the EOS (case of $\mathrm{Cu}$ ) or a negligible one (case of $\mathrm{Fe}$ ). To better highlight the differences we report in Figure 4 the ratio $\alpha=\mathrm{P}$ (Twist boundary) $/ \mathrm{P}$ (bulk) versus bubble radius for the cases studied. For the radius of interest, between 0.5 and $2 \mathrm{~nm}$, the pressure of bubbles in Fe appear insensitive to the location of the bubble, while in $\mathrm{Cu}$, a small decrease, up to $10 \%$ for the small bubbles, is observed at the twist boundaries in both orientations studied. Is this effect relevant to produce a different bubble-to-void transition radius? To answer this question, we have analyzed the elements that affect the bubble pressure.

The most important is the strength of the surrounding matrix. The differences observed between $\mathrm{Cu}$ and Fe might be qualitatively related to the properties of the dislocation networks by noticing that the $\{100\}$ TGB in $\mathrm{Fe}$ is composed of $\langle 100\rangle$ screw dislocations, a high energy dislocation having a 33\% more energy density (line tension) than the $\frac{1}{2}\langle 111\rangle$ dislocation. In $\mathrm{Cu}$ TGBs on the other hand, the cases studied are those with the lowest energy dislocations. The formation energies of vacancies and He atoms depend on the dislocation core properties. 


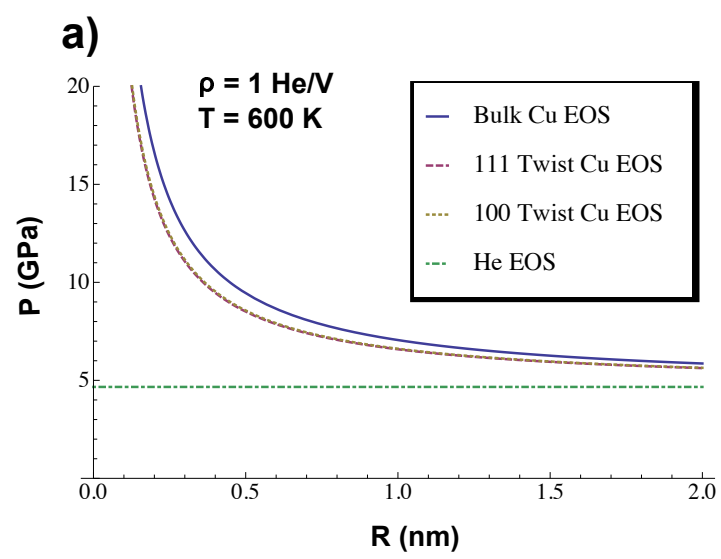

b)

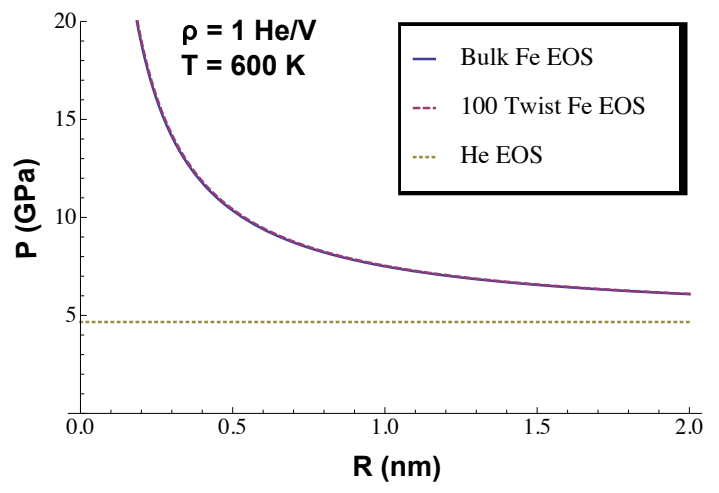

FIG. 3: Pressure vs radius at $\rho=1 \mathrm{He} / \mathrm{Vac}$ and $T=600$ $\mathrm{K}$, (a) for He bubbles located in bulk, at a (100) and at (111) twist boundary in $\mathrm{Cu}$, and (b) for He bubbles located in bulk or at a (100) twist boundary in Fe (lower figure). For comparison, the pressure for He (not in a metal) is also shown at the same density and temperature, as a horizontal line (no R-dependence)

\section{B. Study of $\gamma$ in the bubble at an interface}

To go beyond the qualitative argument and the approximations used in Eqs. 3 and 4, let us analyze more in detail the thermodynamics of this system. First, we need to clarify the reference state used to define the formation energy of a vacancy. For historical reasons, the process of creating a vacancy has been considered as the result of removing an atom from a perfect crystal position and placing it at a step in a surface; with this definition, the formation energy of a vacancy at a surface is zero. When computer simulations become the main tool to calculate these quantities, the definition of formation energy of a vacancy took a different reference state, namely, the removed atom is placed at a reservoir with the chemical potential equal to that of a perfect crystal. With this definition, the formation energy of a vacancy at a free surface is different from zero; in particular, it is less than in bulk, giving a higher equilibrium concentration at the surface than in bulk (see Eq. 2).

Now, if we look at Eq. 4, we see that this expression

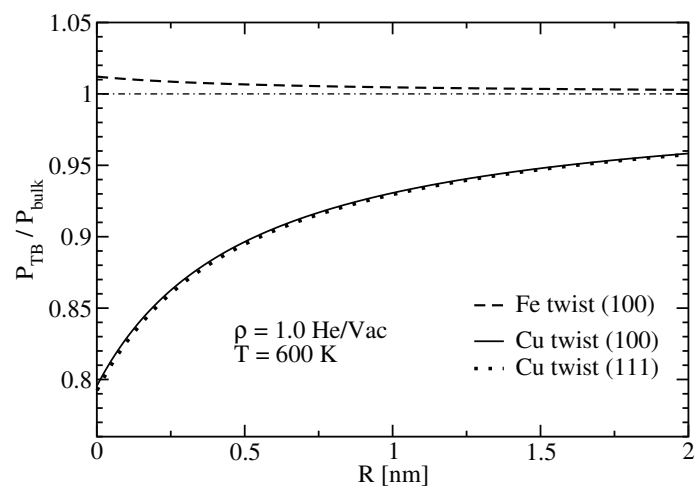

FIG. 4: Ratio $\alpha=\mathrm{P}(\mathrm{GB}) / \mathrm{P}($ bulk) for the interfaces in Fe and $\mathrm{Cu}$, with data from Fig. (4).

for the vacancy concentration assumes that the formation energy of a vacancy at a free surface $(R \rightarrow \infty)$ is equal to its value in bulk. However, in a more precise treatment, this value needs to be specified explicitly. The chemical potential of a vacancy at the bubble boundary in bulk can be therefore written as

$$
\mu_{\mathrm{v}}^{\mathrm{b}}=\mathrm{E}_{\mathrm{v}}^{\mathrm{f}-\text { surf }}+\left(\frac{2 \gamma(\mathrm{R})}{\mathrm{R}}-\mathrm{P}\right) \Omega
$$

where $\mathrm{E}_{\mathrm{v}}^{\mathrm{f}-\text { surf }}$ and $\gamma(R)$ depend on the specific surface and $\gamma$ has an R-dependence ${ }^{15}$. Figure 5 shows the formation energy values for a vacancy around a $1.4 \mathrm{~nm}$ and a $2.8 \mathrm{~nm}$ radius voids in bulk Fe. There is significant dispersion, including sites with close to zero formation energy (note that although small the values are positive and no spontaneous vacancy should be created). Also, in the figure, we report the bulk and surface values $(\{100\}$ and $\{111\}$ ) of the formation energy of the vacancy, and the average value. From the Fe EAM potential by Ackland et al. ${ }^{18}$ used in this study we obtained the vacancy formation energies at the $\{100\}$ and $\{111\}$ surfaces:

$$
\begin{aligned}
& \mathrm{E}_{\mathrm{v}}^{\mathrm{f}-\operatorname{surf}(100)}=0.51 \mathrm{eV} \\
& \mathrm{E}_{\mathrm{v}}^{\mathrm{f}-\operatorname{surf}(111)}=0.81 \mathrm{eV}
\end{aligned}
$$

with surface energies

$$
\begin{aligned}
& \gamma(100)=0.110 \mathrm{eV} / \AA^{2} \\
& \gamma(111)=0.123 \mathrm{eV} / \AA^{2}
\end{aligned}
$$

Both experimentally and theoretically bubbles are observed to nucleate and grow preferentially at interfaces ${ }^{4}$. Figure $6^{19}$ shows a He bubble at a (110) twist boundary in Fe made of two types of dislocations, $\frac{1}{2} a_{0}\langle 111\rangle$ (red) and $a_{0}\langle 100\rangle$ (blue) screws. Monte Carlo simulations show that He bubbles nucleate at the junctions of the network ( $a_{0}\langle 100\rangle$ segments). A schematic of the different vacancy chemical potentials that might be found in the system is also shown (Fig. 6(c)). 

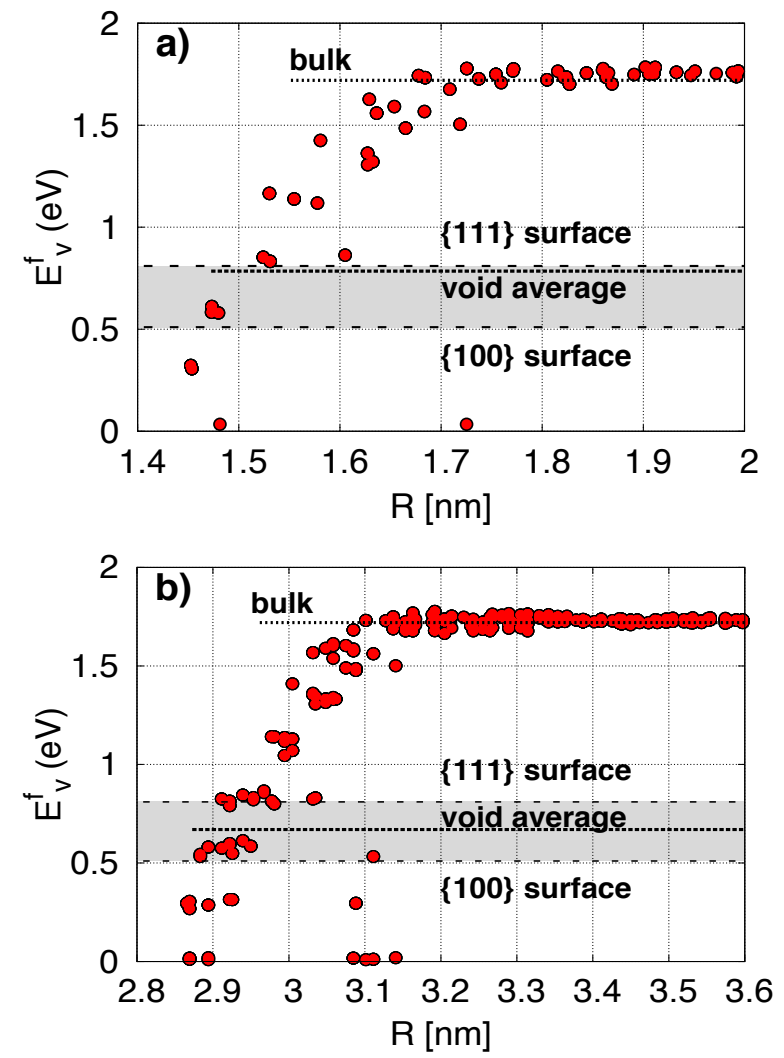

FIG. 5: Formation energy of vacancies at sites close to a void of $3 \mathrm{~nm}$ diameter (a) and $6 \mathrm{~nm}$ diameter (b) radii. Also shown are the values predicted by Eq. 10 at those radii, and the limiting values corresponding to a vacancy in bulk and at a flat surface.
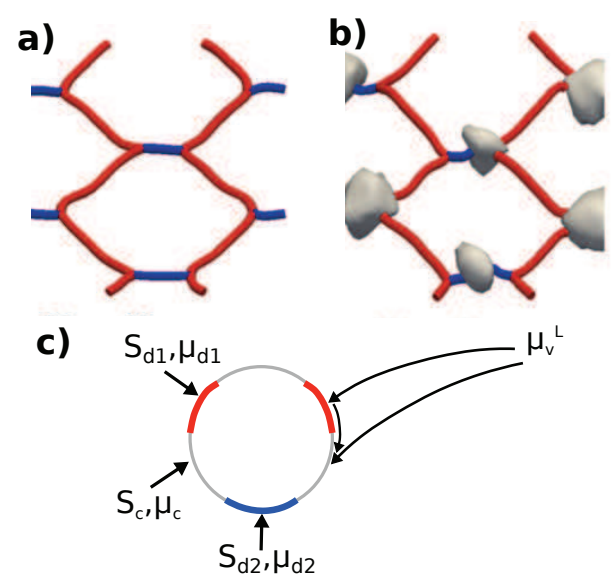

FIG. 6: (a) Dislocation network in a (110) twist boundary in bcc Fe; red indicates screw dislocations with $\frac{1}{2} a_{0}\langle 111\rangle$ Burgers vector, blue shows screw dislocations with $a_{0}\langle 100\rangle$ Burgers vector. (b) Monte Carlo simulations of He loading shows the junctions of the network to be the preferential sites for nucleation. (c) Schematic view of the He bubble as composed of a sphere with patches with different surface energy. $S_{d i}$ and $\mu_{d i}$ represent the area and vacancy chemical potential associated to the dislocation type i.
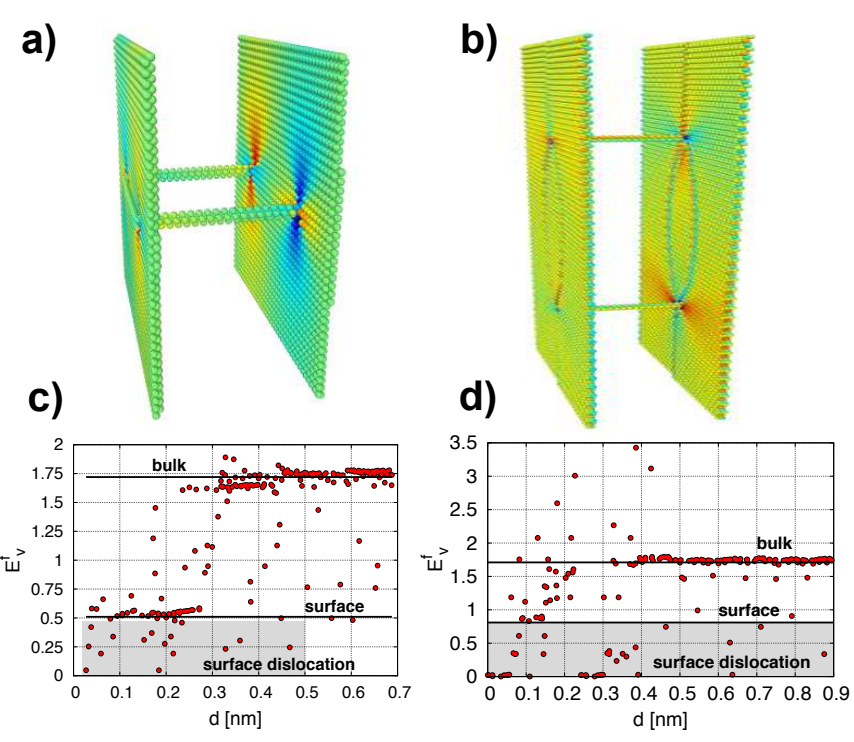

FIG. 7: (a) $a_{0}\langle 100\rangle$ screw dislocations arriving at a (100) surface in Fe. (b) similar for a $\frac{1}{2} a_{0}\langle 111\rangle$ screw dislocation arriving at a (111) surface. (c)-(d) formation energy of vacancies vs distance from the surface. Vacancy formation energy in bulk $(1.72 \mathrm{eV})$ and at the surface $(0.51 \mathrm{eV}$ and $0.81 \mathrm{eV}$ respectively) are indicated by horizontal lines. The shaded area shows sites where the formation energy of the vacancy is less than at a perfect surface, corresponding to dislocation core sites.

For a bubble formed at the interface we hypothesized that the chemical potential can be written as

$$
\mu_{\mathrm{v}}^{\mathrm{d}}=\mathrm{E}_{\mathrm{v}}^{\mathrm{f}-\mathrm{d}} \approx \mathrm{E}_{\mathrm{v}}^{\mathrm{f}-\operatorname{surf} / \mathrm{d}}+\left(\frac{2 \gamma(\mathrm{R})}{\mathrm{R}}-\mathrm{P}\right) \Omega
$$

with $\mathrm{E}_{\mathrm{v}}^{\mathrm{f}-\text { surf/d }}$ the vacancy formation energy at a surface where a perpendicular dislocation impinges and the term $\left(\frac{2 \gamma(\mathrm{R})}{\mathrm{R}}-\mathrm{P}\right) \Omega$ is the same as in bulk. To verify this hypothesis, molecular statics (MS) calculations have been performed to calculate the vacancy formation energies in Fe at the $\{100\}$ and $\{111\}$ surfaces with $a_{0}\langle 100\rangle$ and $\frac{a_{0}}{2}\langle 111\rangle$ perpendicular dislocations impinging each surface, respectively. Figure 7 shows the atomistic models used and the values obtained. Clearly, vacancies at the core, where the dislocation impinges the surface, have the lowest formation energies. In this case, negative formation energy values were found. The atoms sitting where that happens were removed from the system, such that all the remaining values are positive.

The vacancy formation energy at a $\langle 100\rangle$ and $\frac{a_{0}}{2}\langle 111\rangle$ dislocation core in $\mathrm{Fe}$ has been calculated, resulting in $\mathrm{E}_{\mathrm{v}}^{\mathrm{f}-\mathrm{d}\langle 100\rangle}=0.71 \mathrm{eV}$ and $\mathrm{E}_{\mathrm{v}}^{\mathrm{f}-\mathrm{d}\langle 111\rangle}=0.93 \mathrm{eV}$, respectively. Also, the vacancy formation energy at voids of 1.4 and $2.8 \mathrm{~nm}$ radius with the $\langle 100\rangle$ and $\frac{a_{0}}{2}\langle 111\rangle$ dislocations impinging them has been obtained, averaging the values of the sites where the dislocation and the void intersect. The results of these calculations are shown in Fig. 8. Solid points represent the average results obtained 


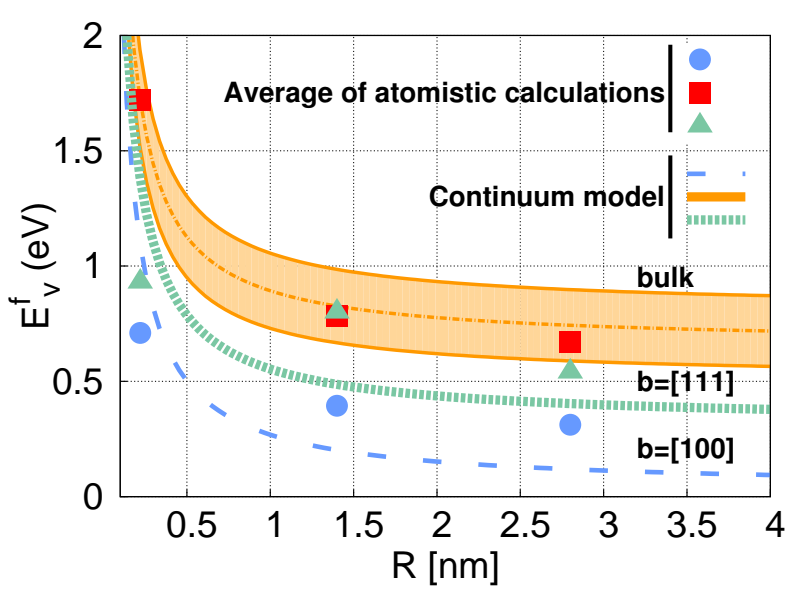

FIG. 8: A continuum approach to the formation energy of a vacancy on the surface of a void, Eq. 10, together with points corresponding to average values of atomic scale calculations.

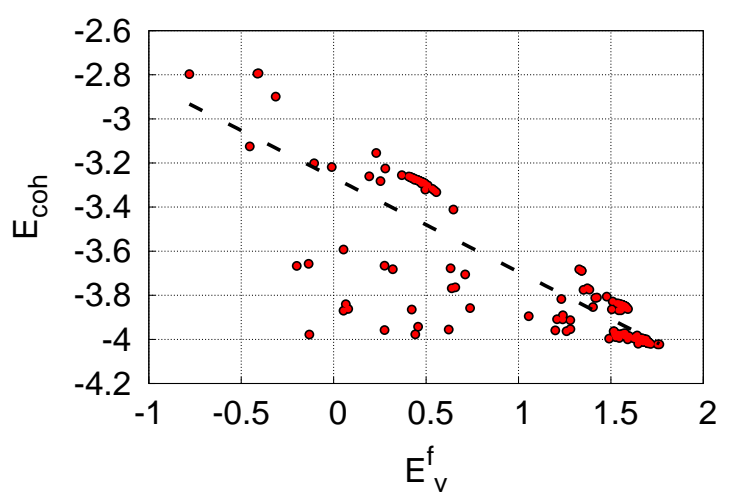

FIG. 9: Relation between the formation energy of a vacancy and the cohesive energy of the atom sitting at the same location. The relation shows that the higher the cohesive energy, the higher the vacancy formation energy.

with MS and the lines show the continuum approach. For bulk we have used the values for both surfaces (in orange) with the dashed line showing an average. In the case where dislocations are present, both the continuum approach and the atomistic data follow the same trends. Although the match is not perfect the qualitative picture is captured by the model in Eq. 10. We note that the more energetic $\langle 100\rangle$ dislocation leads to lower vacancy formation energies compared to the $\frac{a_{0}}{2}\langle 111\rangle$.

To make this information usable in a quantitative expression for different GBs, we characterize the relation between the type of dislocation and the formation energy of a vacancy at a surface where the dislocation core impinges, by using two simplifying assumptions. The first relates the cohesive energy of an atom to the formation energy of a vacancy at that site. Figure 9 shows such relation for the data reported in Fig. 7; from it we obtain that $\mathrm{E}_{\mathrm{v}}^{\mathrm{f}}=7.65 \mathrm{eV}+2.3 \mathrm{E}_{\mathrm{coh}}$. The second assumption is that the cohesive energy of an atom at the core of a

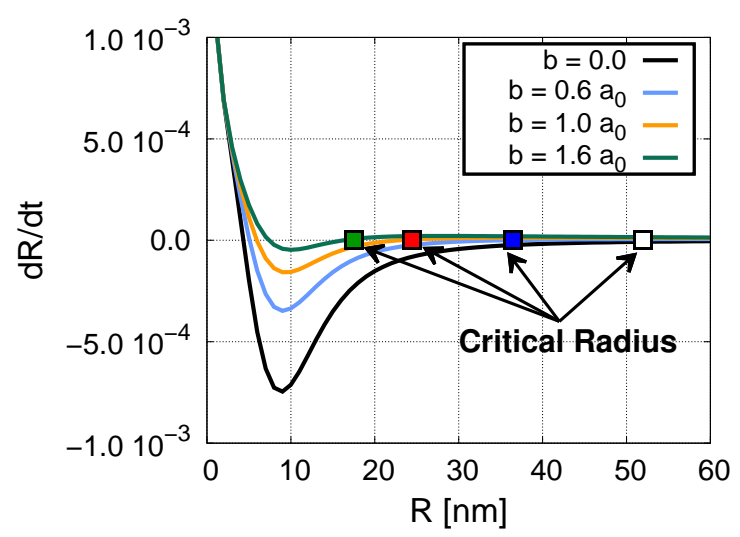

FIG. 10: Variation of the stable and critical radii for the bubble to void transition for bubbles sitting at twist boundaries dislocations with different magnitude of the Burgers vector.

dislocation is proportional to the core energy of the dislocation, namely $\mathrm{E}_{\mathrm{coh}}=\mathrm{E}_{\mathrm{coh}}^{0}-\alpha \mathrm{Gb}^{2}$ with $\alpha$ a positive constant, $\mathbf{G}$ the shear modulus, and $\mathbf{b}$ the magnitude of the Burgers vector. Both assumptions are strictly valid if the cohesive energy of the solid is given by a quasichemical model. We arrive at the final expression for the vacancy chemical potential at the normal surface region and at the dislocation patch region of a bubble, namely,

$$
\begin{gathered}
\mu_{\mathrm{v}}^{\mathrm{b}}(\mathrm{R})=\mathrm{E}_{\mathrm{v}}^{\mathrm{f}-\text { surf }}+\left(\frac{2 \gamma}{\mathrm{R}}-\mathrm{P}\right) \Omega \\
\mu_{\mathrm{v}}^{\mathrm{d}}(\mathrm{R}, \mathrm{b})=\mathrm{E}_{\mathrm{v}}^{\mathrm{f}-\text { surf }}-\mathrm{ab}^{2}+\left(\frac{2 \gamma}{\mathrm{R}}-\mathrm{P}\right) \Omega
\end{gathered}
$$

From our calculations for $\mathrm{Fe}\langle 100\rangle$ and $\langle 111\rangle$ screw dislocations, $a=0.17 \mathrm{eV} / \AA^{2}$. Equating these chemical potentials to $\mu_{v}^{L}$ we obtain the vacancy concentrations and replacing these values into Eq. 1 we get the expression for the bubble growth rate as

$$
\begin{aligned}
& \frac{\mathrm{dR}_{\mathrm{c}}}{\mathrm{dt}}= \\
& \frac{\Omega}{\mathrm{R}}\left[\mathrm{Z}_{\mathrm{v}}^{\mathrm{c}} \mathrm{D}_{\mathrm{v}} \mathrm{C}_{\mathrm{v}}-\mathrm{Z}_{\mathrm{i}}^{\mathrm{c}} \mathrm{D}_{\mathrm{i}} \mathrm{C}_{\mathrm{i}}-\mathrm{D}_{\mathrm{v}}\left(\mathrm{Z}_{\mathrm{v}}^{\mathrm{c}} \mathrm{C}_{\mathrm{v}}^{\mathrm{c}}(\mathrm{r})+\sum_{\mathrm{i}} \mathrm{Z}_{\mathrm{d}_{\mathrm{i}}} \mathrm{C}_{\mathrm{d}_{\mathrm{i}}}(\mathrm{b})\right)\right]
\end{aligned}
$$

To see the effect of dislocations on the bubble to void critical radius, we plot Eq. 13 for different Burgers vectors, corresponding to possible TGBs in Fe and assume that the first and second terms of this equation remain constant. Figure 10 shows that the critical radius strongly depends on b, while the stable radius just weakly depends on it. Note that the stronger the sink the lower the critical radius as the flux of vacancies is larger. However, the sink distribution can lead to a lower probability to reach the critical radius than in bulk. Although the value of the different parameters might change depending on the He density, the qualitative picture given by the model will be valid. 


\section{CONCLUSIONS}

The results discussed in this work, together with our previous work on equilibrium bubbles at the nanoscale ${ }^{15}$, provide a picture of the rich behavior of nanoscale $\mathrm{He}$ bubbles in $\mathrm{Cu}$ and $\mathrm{Fe}$, in bulk and at grain boundaries. In particular, in this work we provide a quantitative expression validated with atomistic calculations to assess the role of different types of grain boundary dislocations on the vacancy chemical potential, that leads to different bubble growth rates. We analyzed how the critical size for the bubble to void transition depends on the Burgers vector of different dislocation types. Concisely, grain boundary dislocations having large Burgers vector displace the bubble to void transition to lower radii compared to dislocations with smaller Burger vectors.

\section{ACKNOWLEDGMENTS}

This work was performed by the Center for Materials at Irradiation and Mechanical Extremes, an Energy Frontier Research Center funded by the U.S. Department of Energy (Award Number 2008LANL1026) at Los Alamos National Laboratory. This research used resources provided by the LANL Institutional Computing Program. LANL, an affirmative action/equal opportunity employer, is operated by Los Alamos National Security, LLC, for the National Nuclear Security Administration of the U.S. DOE under contract DE-AC52$06 \mathrm{NA} 25396$.
* Electronic address: enriquem@lanl.gov

${ }^{1}$ G. R. Odette, M. J. Alinger, B. D. Wirth, Recent developments in irradiation-resistant steels, Ann. Rev. Mater. Res. 38 (2008) 471.

2 P. A. Thorsen, J. B. Bilde-Sorensen, B. N. Singh, Influence of grain boundary structure on bubble formation behaviour in helium implanted copper, Mat. Sci. Forum 207209 (1996) 445.

${ }^{3}$ P. A. Thorsen, J. B. Bilde-Sorensen, B. N. Singh, Bubble formation at grain boundaries in helium implanted copper, Scripta Mater. 51 (2004) 557-560.

4 J. Hetherly, E. Martinez, Z. F. Di, M. Nastasi, A. Caro, Helium bubble precipitation at dislocation networks, Scripta Mater. 66 (2012) 17-20.

5 M. J. Demkowicz, A. Misra, A. Caro, The role of interface structure in controlling high helium concentrations, Cur. Op. Sol. State Mat. Sci. 16 (2012) 101-108.

${ }^{6}$ W. Z. Han, M. J. Demkowicz, N. A. Mara, E. G. Fu, S. Sinha, A. D. Rollet, Y. Q. Wang, J. S. Carpenter, I. J. Beyerlein, A. Misra, Design of radiation tolerant materials via interface engineering, Adv. Mat. 25 (2013) 6975-6979.

7 G. S. Was, Fundamentals of Radiation Materials Science, Springer, 2007.

${ }^{8}$ L. K. Mansur, Effects pf point defect trapping and soulte segregation on irradiation-induced swelling and creep, J. Nucl. Mater. 83 (1979) 109-127.

9 L. K. Mansur, W. A. Coghlan, Mechanisms of helium interaction with radiation effects in metals and alloys: A review, J. Nucl. Mater. 119 (1983) 1-25.
10 L. K. Mansur, E. H. Lee, P. J. Maziasz, A. P. Rowcliffe, J. Nucl. Mater. 633 (1986) 141.

11 L. K. Mansur, Theory and experimental background on dimensional changes in irradiated alloys, J. Nucl. Mater. 216 (1994) 97-123.

12 L. K. Mansur, The reaction rate theory of radiation effects, JOM 48 (1996) 28.

13 R. E. Stoller, G. R. Odette, Analytical solutions for helium bubble and critical radius parameters using a hard sphere equation of state, J. Nucl. Mater. 131 (1985) 118.

14 A. Caro, D. Schwen, E. Martinez, Structure of nanoscale gas bubbles in metals, Appl. Phys. Lett. 103 (213115).

15 A. Caro, D. Schwen, J. Hetherly, E. Martinez, The capillarity equation at the nanoscale: Gas bubbles in metals, Acta Mater. 89 (2015) 14-21.

16 R. C. Tolman, The effect of droplet size on surface tension, J. Chem. Phys. 17 (1949) 333.

17 J. D. Eshelby, The determmination of the elastic field of an ellipsoidal inclusion and related problems, J. D. Proc. of the Royal Soc. of London Series A-Mathematical and Phys. Sci. 241 (1957) 376-396.

18 G. J. Ackland, M. I. Mendelev, D. J. Srolovitz, S. Han, A. V. Barashev, Development of an interatomic potential for phosphorus impurities in alpha-iron, J. Phys: Condens. Matter 16 (2004) 2629-2642.

19 N. N. Kumar, E. Martinez, B. K. Dutta, G. K. Dey, A. Caro, Nodal effects in $\alpha$-iron dislocation mobility in the presence of helium bubbles, Phys. Rev. B 87 (2013) 054106. 